\title{
Environmental Self-Auditing: \\ Setting the Proper Incentives for Discovering and Correcting Environmental Harm
}

by

\begin{abstract}
Alexander S.P. Pfaff, Columbia University
Chris William Sanchirico, Columbia University
\end{abstract}

January 1997, Revised February 1998

Discussion Paper Series No. 9798-10

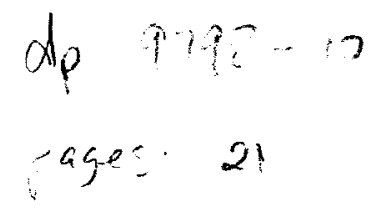




\title{
ENVIRONMENTAL SELF-AUdiTING:
}

\author{
SetTing THe Proper InCENTIVes FOR
}

\section{DISCOVERING AND CORRECTING ENVIRONMENTAL HARM ${ }^{*}$}

\author{
Alexander S. P. Pfaff \\ Columbia University, Department of Economics \\ and Center for Environmental Research and Conservation \\ Chris William Sanchirico \\ Columbia University, Department of Economics and School of Law
}

January 1997 (Minor Revisions: 4/3/97, 2/2/98)

\section{Preliminary and Incomplete}

\footnotetext{
"We thank the Center for International Business Education at Columbia University for financial support. We also thank Barry Adler, Jennifer Arlen and Reinier Kraakman for helpful comments.
} 


\section{INTRODUCTION}

Since the 1970's, the amount of environmental regulation at all levels of government has increased significantly. Major federal statutes include the Clean Air Act, Federal Water Pollution Act, Resource Conservation and Recovery Act (RCRA), Toxic Substances Control Act (ToSCA) and the Comprehensive Environmental Response, Compensation, and Liability Act (CERCLA). In addition, states have their own environmental laws and regulations. The resulting web of often highly technical requirements makes it difficult for even the regulated enterprise itself to know whether it is in compliance with applicable law. In response, many firms have instituted a policy of conducting their own "environmental audits."

Despite the increased incidence of such audits many commentators have suggested that the practice is still far rarer than it should be. ${ }^{2}$ Self-auditing is regarded as more thorough and efficient than periodic audits by the regulator. Yet, firms are reportedly reluctant to conduct such audits for fear that the information they gather on compliance problems will be obtained by the government and/or adverse private parties (presumably through discovery, subpoena or whistleblowing) and used against them in a subsequent proceeding. By carefully investigating their own compliance status, the argument goes, firms are effectively aiding in their own prosecution and/or adverse suit. ${ }^{3}$

\footnotetext{
${ }^{1}$ See, e.g., Kirsch and Viers (1996).

${ }^{2}$ See, e.g., Cooney et. al. (1995c) ("Environmental auditing is not used as frequently as it should be, however, due in part to governmental reluctance to give formal protection to internal documents generated during the audit process.")

${ }^{3}$ See, e.g., ibid. and Moore and Newkirk (1995), ("Substantial disincentives to self evaluation have existed because of enforcement risk associated with penalties imposed when noncompliance is found and reported and the fear that
} 
Since 1986 the EPA has taken a series of tentative steps to address this concern, cumulating in a December 1995 "Policy Guideline." Reduced to its essential economic content, the guideline lowers fines ${ }^{5}$ for firms that discover violations through a program of self-auditing, $\underline{\text { o }}$ long as the firm voluntarily discloses these violations and then promptly corrects the problem. Industry sentiment is that the EPA policy does not go far enough and legislation is now pending in Congress which would provide more sweeping protections. Stronger protections have in fact already been passed in several states, notably Oregon and Colorado. ${ }^{6}$ More than ten states ${ }^{7}$ in all have enacted into law some form of evidentiary "audit privilege" for the information revealed by self-audit; ${ }^{8}$ others also offer immunity for harms discovered and fixed.

The question of how best to treat self-auditing is in essence a two-tiered incentive problem. As in the usual torts problem, ${ }^{9}$ the primary concern is inducing firms to take appropriate levels of "environmental care" through a system of fines or charges probabilistically applied via random and imperfect auditing. But in a technically and legally complicated regulatory regime, it is also necessary to induce firms to carefully figure out whether, where and how to apply that care effort. The difficulty, according to all reports, is that such self-investigation effectively improves the regulator's own auditing system and so increases the frequency that fines used to induce care effort will be assessed.

the information will be used against the company."). Feeley (1995) ("A self-audit can become a "prosecutorial road map,' allowing disclosure to be used as an enforcement tool.")

${ }^{4}$ EPA (1995).

${ }^{5}$ Through both the explicit reduction of the "gravity" component of civil fines and the (non-binding) promise not to prosecute based on self-discovered violations.

${ }^{6}$ See, e.g. Kirsch and Viers (1996).

${ }^{7}$ These are Arkansas, Colorado, Idaho, Illinois, Indiana, Kansas, Kentucky, Mississippi, Oregon, Utah, Virginia, Wyoming. At least 25 other states have considered similar legislation. 
This paper applies the existing legal/economic analysis of torts to the two-tiered incentive issued raised by the self-auditing issue. We find that conventional tort remedies based on harm and/or "care" (strict liability and negligence with punitive add-ons to reflect the possibility that firms are not caught in violation) fail to produce the socially optimal amount of self-auditing. To fix the problem we propose that punitive fines be reduced for firms that conduct their own investigation. This echoes Arlen's (1994) earlier findings with respect to corporate criminal liability. (See also the contemporaneous, Arlen and Kraakman (1997)).

Importantly, we suggest this reduction be made regardless of whether the firm voluntarily discloses the results of its investigation, and indeed regardless of whether the firm has chosen to fix the problems it finds. This is in marked contrast to the EPA policy now in effect, which holds voluntary disclosure and corrective action as preconditions for fine reduction; and which, through additional criminal prosecution based in part on a knowledge element, ${ }^{10}$ essentially punish the firm more when it knows of an unfixed problem than when it is ignorant.

We also consider whether making fines contingent on voluntary disclosure (rather than on investigation directly) could be used to correctly align the incentives for self-auditing. We find that the answer is yes, but only to the extent that fine reductions for disclosure may be used to effectively condition fines on investigation (this is explained within). Again in contrast to EPA policy, this requires, among other things, that fine reduction for disclosed violation not be made contingent on whether the violation was corrected.

\footnotetext{
${ }^{8}$ There is general agreement among legal scholars that traditional privileges (including attorney client, work product and the self-evaluative privilege) fail to cover this case.

${ }^{9}$ See, e.g. Shavell (1980) and Polinsky and Rubinfeld (1988).

${ }^{10}$ Note, however, that the usual "knowledge" requirement is generally not as strict for environmental crimes. See, e.g. Cooney et. al. (1995b).
} 
The paper proceeds as follows: Section 2 sketches a simple model. Section 3 starts by identifying the socially optimal solution to the two-tiered choice of investigation and fixing. It turns next to the failure of conventional strict liability and negligence rules to attain this optimum. Next, it proposes a solution involving conditioning fines on investigation. Lastly, it shows that if investigation is verifiable, it is possible to obtain effectively the same solution by conditioning on disclosure of investigation rather than investigation itself.

\section{A Simple Model}

A firm is uncertain about the amount of environmental harm $h$ that its operation in the current (and only) period will cause. It is uncertain about both the magnitude and nature of this harm. Without understanding the nature of the harm, the firm cannot take corrective action. Its beliefs over magnitude are given by the probability measure $P$.

In order to discover the magnitude and nature of the harm the firm must conduct an environmental self-audit (henceforth an "investigation"). We assume that the firm decides only whether or not to investigate, not how much or how to investigate. We also assume that if the firm investigates it learns with certainty both the magnitude and nature of the harm. It costs the firm $i$ to investigate.

If the firm chooses to investigate, it then decides whether or not to fix the problem(s) that it finds. The fixing decision is also binary and if the firm fixes the problem there is no residual harm. The cost of alleviating the harm depends on the magnitude of the harm and is denoted $c_{h}$. 
Left to itself, the firm will neither investigate nor fix since these are costly actions and it does not care about the harm it is causing. The second actor in our model, a regulatory agency, considers not only private costs, but also the harm itself. With some probability depending on the harm itself, the agency learns the magnitude and nature of the harm caused by the firm. In accord with rationales presented in the popular press for special treatment of voluntary disclosure, ${ }^{11}$ we assume that this probability is always larger if the firm has itself investigated. Perhaps the existence of a "paper trail" makes it more likely that, conditional on auditing, the agency learns of the harm. If the firm has investigated, the probability that the government learns the harm is $\bar{\beta}_{h}$. If it has not, the probability is $\underline{\beta}_{h}<\bar{\beta}_{h}{ }^{12}$

\section{Consideration of Alternative Policies}

We begin by characterizing what investigation and fixing decisions the regulatory agency would make if it controlled the firm. We then consider alternative fine structures that the agency might use in order to align private incentives with public interest. ${ }^{13}$ These analyses are presented in two steps, working backwards: first the decision to fix, conditional upon having investigated, is considered; next the initial decision to investigate is analyzed.

\footnotetext{
"See footnote 3, supra.

${ }^{12}$ These probabilities are a composite of 1) the probability that the regulatory agency audits the firm and 2) given that the agency has audited, the probability that the agency finds the harm. We abstract from the possibility that the agency either finds only part of the harm or overstates the harm. If it finds harm at all, it finds the right level. ${ }^{13}$ In fact we are considering only a sub-problem of a more general social welfare problem. In principle, the optimal probabilities of government spot-auditing would be derived from a model in which the government cost of spotauditing and the firms cost of self-auditing were included. Here we take these probabilities as exogenously given and focus on the issue of how fines should be structured.
} 


\subsection{Society's Problem}

What contingent plan for investigation and fixing is optimal for society? Having investigated and found harm of magnitude $h$, fixing is a best choice if and only if

$$
c_{h} \leq h .
$$

This produces an optimal fixing decision contingent on $h$. Given this best contingent plan, investigation imposes expected social costs of

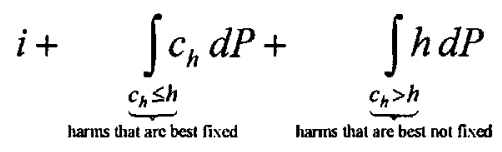

On the other hand, not investigating imposes expected social costs of

$$
\int h d P
$$

Combining (2) and (3), it is best for society to investigate if and only if

$$
\underset{\text { direct costs }}{i} \underbrace{\int_{c_{h} \leq h} h-c_{h} d P}_{\text {benefits }} .
$$

The social costs of investigation (as we do the accounting here) are the direct costs $i$ of conducting the search for harm. The social benefits are the savings $\left(h-c_{h}\right)$ in all the cases where we fix, having discovered the harm (i.e. all $h$ with $c_{h} \leq h$ ).

In order for our firm to make the optimal decision for society we must employ a fine structure that induces the firm to make the decision to fix, having investigated, according to (1) and the decision to investigate according to (4). 


\subsection{Conventional Strict liability}

We consider first a policy of strict liability (with additional punitive damages to account for the probability that the firm is not caught). While this can succeed (unsurprisingly) in giving the firm the right incentives to fix conditional upon having investigated, we find that it results in too little investigation. As we clarify below, there are in fact two distinct distortions to the investigation decision (given that incentives in the post-investigation fixing decision are correctly aligned). The first is born from the fact that investigation increases the probability of being fined should the firm choose not to fix the harm that it finds. The second is due to the firm's undervaluing the benefits of investigating in cases where it does fix.

Under a strict liability standard, the firm, if found out, must pay a fine based on harm caused, with no regard to the relative size of fixing costs and harm. Clearly, a purely compensatory fine will not create the proper incentives for fixing, following investigation. That is, if we only charge the firm for the harm it causes whenever we catch it, the firm will decide whether to fix based on $c_{h} \leq \bar{\beta}_{h} h$, which will in general mean that the firm will fix too infrequently, as compared to (1). So let us immediately jump to a compensatory fine plus a punitive component necessary to align fixing incentives. It is easy to see that the total fine $F_{h}$ (both compensatory and punitive elements) for harm $h$ must be such that $\bar{\beta}_{h} F_{h}=h$. Here $h$ itself is the compensatory component of $F_{h}$, while the punitive component is $G_{h}=\frac{h}{\bar{\beta}_{h}}-h$.

Turning now to the investigation decision, investigation imposes expected costs on the firm

of

$$
i+\int_{c_{h} \leq \bar{\beta}_{h} F_{h}} c_{h} d P+\int_{c_{h}>\bar{\beta}_{h} F_{h}} \bar{\beta}_{h} F_{h} d P .
$$


Note that when the firm does not fix $\left(c_{h}>\bar{\beta}_{h} F_{h}\right)$ it faces the probability $\bar{\beta}_{h}$ that it will be fined. When it does fix, there is no possibility of a fine, since by our simplifying assumption, the harm is eliminated.

Not investigating imposed expected costs of

$$
\int \underline{\beta}_{h} F_{h} d P
$$

Note that the probability of being fined is lower in (6) than in (5) due to the fact that firm has not investigated in (6). Combining (5) and (6), the firm investigates if and only if

$$
i+\int_{c_{h}>\bar{\beta}_{h} F_{h}}\left(\bar{\beta}_{h}-\underline{\beta}_{h}\right) F_{h} d P \leq \int_{c_{h} \leq \bar{\beta}_{h} F_{h}} F_{h} F_{h}-c_{h} d P .
$$

Selectively substituting $\bar{\beta}_{h} F_{h}=h$ yields

$$
i+\underbrace{\int_{c_{h} h}\left(\bar{\beta}_{h}-\underline{\beta}_{h}\right) F_{h} d P}_{\text {Term A }} \leq \underbrace{\int_{c_{h} \leq h} \underline{\beta}_{h} F_{h}-c_{h} d P}_{\text {Term B }} .
$$

We see that this criterion differs from society's investigation decision in (4) on two scores. Term A in (8) concerns cases in which the harm would not be fixed if found. Its presence in (8) and not in (4) reflects the fact that investigation exposes the firm to a higher probability of being caught. This disincentive to investigation seems to be what firms themselves point to in arguing for special treatment of environmental self-audits.

The second discrepancy in investigation criteria has received much less, if any, attention in the popular press. This discrepancy, namely the difference between Term B in (8) and the right hand side of (4), concerns harms which would be fixed once revealed by investigation. In these 
cases the firm compares the cost of fixing $c_{h}$ to the expected fine conditional on not

investigating. The discrepancy arises because this expected fine is calculated using $\underline{\beta}_{h}$, the

probability of being caught having not investigated. Since the fine $F_{h}$ was set so that $\bar{\beta}_{h} F_{h}=h$, it must be that $\underline{\beta}_{h} F_{h}<h$ and so the firm's net benefit from investigation in these cases falls short of society's. This effect then, also weakens the incentive to investigate.

\subsection{Conventional Negligence}

As noted above, one of the problems of strict liability is that when the firm investigates but chooses not to fix, its investigation increases the probability of getting caught, adding an unwanted disincentive to investigation. If fixing incentives are properly aligned, this problem only arises when it is best for the firm, as well as society, not to fix. Thus a negligence standard, which only imposes fines in cases where society would have fixed, might be expected to align public and private incentives to investigate. Recall though that there are two potential discrepancies between the public and private incentives for investigation. While a negligence standard does eliminate the discrepancy in incentives in the cases in which neither the firm nor society would choose to fix, it fails to do so where the firm should and would fix.

To show this formally, we begin by defining the negligence standard. We set

$$
F_{h}=\left\{\begin{array}{ll}
\frac{h}{\bar{\beta}_{h}} & \text { if } c_{h} \leq h \\
0 & \text { if } c_{h}>h
\end{array} .\right.
$$

By standard arguments, this negligence standard creates the proper incentives to fix having investigated. If $c_{h} \leq h$, the firm faces an expected fine of $\bar{\beta}_{h} F_{h}=h$ if it fails to fix. Hence, it fixes. If, on the other hand, $c_{h}>h$, it faces no fine for not fixing and so does not fix. 
Stepping back to the investigation decision, investigating yields expected costs of

$$
i+\int_{c_{h} \leq h} c_{h} d P+\underbrace{\int_{c_{h}>h} 0 d P .}_{\text {not negligent }}
$$

If the firm does not investigate, expected costs are

$$
\underbrace{\int_{c_{h} \leq h} \underline{\beta}_{h} F_{h} d P}_{\text {cases where negligent }} .
$$

Combining (10) and (11), we see that the firm investigates if and only if

$$
i \leq \underbrace{\int_{c_{h} \leq h} \underline{\beta}_{h} F_{h}-c_{h} d P .}_{\text {Term C }}
$$

We see then that Term A from (8) has disappeared as noted above: since there are no fines for investigating and then making the socially optimal decision not fix, the firm is not exposing itself to a greater likelihood of getting caught in these cases. However, Term C in (12) is the same as Term B in we (8) and so we face the same discrepancy between public and private investigation incentives for harms which the firm would choose to fix.

\subsection{Conditioning Fines on Investigation ${ }^{14}$}

What sort of fine structure will completely align public and private incentives? A basic intuition is generated through inspection of the discrepancies between the public and private investigation criteria as laid above. Both the discrepancies that we identify (see Terms A and B in (8)) arise because the firm faces different expected fines depending on whether or not it investigates. This is due to the fact that investigation raises the probability of getting caught 
from $\underline{\beta}_{h}$ to $\bar{\beta}_{h}$. But this can be counteracted by conditioning the fine itself on whether the firm has investigated-in particular, by raising the fine in cases where it is less likely to be imposed. In effect, the agency ends up rewarding the firm for having investigated: the firm is compensated for having helped the agency discover the firm's own violations (through the fact that investigation raises the probability of being caught).

The reader may ask: why should I believe that investigation is observable by the regulator? But this is not the precise issue. The issue is whether the regulator knows of the investigation in cases where the existence of that investigation helped it uncover the firm's noncompliance. ${ }^{15}$

Observability in these cases seems not only plausible, but logically required. If the agency's observation is what causes the incentive problem in the first place, there can be no observability issue in fixing that problem.

Our finding that the regulator should condition fines on investigation per se has interesting implications for policy. As noted in the introduction, corporations and others point to increased exposure to fine and liability as a strong disincentive to self-auditing. Policy solutions suggested (and sometimes implemented) effectively reduce the punitive component of fines for violations uncovered in self-auditing that are voluntarily disclosed and promptly corrected. Our proposal to

\footnotetext{
${ }^{14}$ The analysis in this section is done in the context of strict liability. A similar analysis applies to negligence.

${ }^{15}$ A more complicated model would allow for states of world in which the firm's investigation is only partially (if at all) discovered and/or used by the regulator. By the same logic as in our model, the regulator can still alleviate the disincentive to self-investigation by crediting the firm for the extent to which the agency has actually relied upon the firm's self-investigation to catch the violation. Almost by definition, the agency can always observe the extent to which the firm's investigation helped its own.
} 
reduce fines is contingent only upon the agency's finding that there was investigation, regardless of whether the findings are disclosed and/or corrected. ${ }^{16}$

Returning to the formal model, let $\bar{F}_{h}$ be the fine in the case in which the firm has investigated and let $\underline{F}_{h}$ be the fine when the firm has not investigated. Set $\bar{\beta}_{h} \bar{F}_{h}=\underline{\beta}_{h} \underline{F}_{h}=h$, implying that $\bar{F}_{h}<\underline{F}_{h}$. Incentives to fix having investigated are then properly aligned as above. The cost of investigating is

$$
i+\int_{c_{h} \leq \bar{\beta}_{h} \bar{F}_{h}} c_{h} d P+\int_{c_{h}>\bar{\beta}_{h} \bar{F}_{h}} \bar{\beta}_{h} \bar{F}_{h} d P
$$

The cost of not investigating is

$$
\int \underline{\beta}_{h} \underline{F}_{h} d P
$$

Combining yields the analogy to (7)

$$
i+\int_{c_{h}>\bar{\beta}_{h} \bar{F}_{h}}\left(\bar{\beta}_{h} \bar{F}_{h}-\underline{\beta}_{h} \underline{F}_{h}\right) d P \leq \int_{c_{h} \leq \bar{\beta}_{h} \bar{F}_{h}} \underline{\beta}_{h} \underline{F}_{h}-c_{h} d P
$$

which since $\bar{\beta}_{h} \bar{F}_{h}=\underline{\beta}_{h} \underline{F}_{h}=h$ reduces to (4),

$$
i \leq \underbrace{i}_{\text {benefits }} \leq \underbrace{\int_{c_{h} \leq h} h-c_{h} d P .}_{\text {direci costs }}
$$

\footnotetext{
${ }^{16}$ Note that in the EPA guidelines, fixing is always required for fine reduction, while our model depending on the harm society may prefer not to fix (when the costs of correction exceed the cost of the harm). This difference may reflect the long-standing mandate in Federal policy to not consider the costs of correction.
} 


\subsection{A Model of Voluntary Disclosure}

Is it possible to reconcile our proposal in the previous subsection with EPA policy? In this section we take a step toward answering that question by considering the case in which fines are conditioned on the firm's disclosure of its investigation, rather than investigation itself. (Recall that EPA conditions fine reduction on disclosure and correction). We find that conditioning on disclosure can produce proper incentives, but we emphasize from the start three reasons why this finding does not support the EPA approach.

First, conditioning on disclosure raises difficult issues of credibility: firms may have an incentive to falsify or dissemble in reporting their results. Our mode of analysis here is to give disclosure conditioning its "best chance" by assuming that information disclosed by the firm to the agency is fully verifiable. Importantly, this will mean that the firm can not pretend to have investigated, nor can it shade reports of investigation actually conducted. ${ }^{17}$

Secondly, the reason that disclosure conditioning can be effective is that it can be manipulated in such a way that what is really being conditioned on is, once again, investigation per se, as in our previous proposal. (The formal analysis below explains precisely how.)

Thirdly, our solution in this subsection conditions solely on disclosure and not also on corrective action, as does actual EPA policy. The agency reduces fines for an admission of harm, even when the firm has no intention of correcting that harm.

To make these points precise we modify our model slightly to account for the firm's decision to disclose. Now, after having investigated, the firm chooses not only whether to correct the harm but also whether to report the results of its investigation to the agency. As 
noted, we assume that this report is verifiable, hence veritable. Thus, if the firm finds harm of $h$, and decides to make disclosure to the agency, it is compelled to report " $h$. ." In particular, if it has not investigated, it has nothing to disclose and so the disclosure choice, like the fixing choice, arises only after investigation. ${ }^{18}$ We also assume that such disclosure imposes no direct costs on the firm.

Since the issue here is the effect of conditioning on disclosure, we allow the agency to set two fines: $F_{h}^{T}$ if the firm discloses harm $h$ and $F_{h}^{N}$ if the agency finds out on its own that the harm is $h$. Importantly, the agency does not condition directly on investigation and so $F_{h}^{N}$ is assessed both in the case in which the firm has investigated, not fixed and not disclosed and the case in which the firm has not investigated (and so neither fixed ${ }^{19}$ nor disclosed).

Proceeding backwards as in previous subsections, suppose that the firm has investigated. It has four choices. It can 1) fix and disclose, 2) fix and not disclose, 3) not fix and disclose, or 4) not fix and not disclose. Whether or not the firm discloses, fixing yields direct costs of $c_{h}$ (as disclosure itself is costless). This handles alternatives 1) and 2). If the firm chooses alternative 3), not fixing but disclosing, the firm is fined $F_{h}^{T}$. Finally, alternative 4), not fixing and not disclosing results in an expected fine of $\bar{\beta}_{h} F_{h}^{N}$.

In deciding whether to fix, the firm compares the uniform payoffs from fixing (i.e. alternatives 1) and 2)) to the better of the payoffs from not fixing (i.e. alternatives 3 ) and 4). The

\footnotetext{
${ }^{17}$ Compare this observability issue to that in the previous subsection. There the (non)issue was whether the agency could observe the extent to which the firm's investigation helped it uncover violations. Here the very real issue is whether the agency can verify the truth of the firm's disclosure.

${ }^{18}$ Once again, we make this assumption not because we think it is plausible, but because the points we wish to make about disclosure are sentences of the form, "even if disclosure is verifiable,..."
} 
effective fine from not fixing is then the minimum of fines in 3) and 4) and the firm fixes if and only if

$$
c_{h} \leq \min \left\{F_{h}^{T}, \bar{\beta}_{h} F_{h}^{N}\right\} \equiv m_{h}
$$

Stepping backwards to the investigation decision, and making use of our definition of $m_{h}$, investigation imposes on the firm expected costs of

$$
i+\int_{c_{h} \leq m_{h}} c_{h} d P+\int_{c_{h}>m_{h}} m_{h} d P,
$$

while not investigating imposes costs of

$$
0+\int \underline{\beta}_{h} F_{h}^{N} d P
$$

Combining, we see that the firm will investigate if and only if:

$$
i+\underbrace{\int_{c_{h}>m_{h}} m_{h}-\underline{\beta}_{h} F_{h}^{N} d P}_{\text {Term D }} \leq \underbrace{\int_{c_{h} \leq m_{h}} \underline{\beta}_{h} F_{h}^{N}-c_{h} d P}_{\text {Term E }}
$$

Once again our objective is to set the fines so that the firm's decision to investigate and fix resembles society's. Aligning fixing incentives requires setting fines so that $m_{h}=h$.

Investigation incentives are aligned as well if we also set $\underline{\beta}_{h} F_{h}^{N}=h$, for then Term D disappears and Term E resembles the right hand side of (4). Now, if $m_{h}$ and $F_{h}^{N}$ were independent variables, we could do this without further thought. However, $m_{h}$ depends in principal on $F_{h}^{N}$ and our analysis thus requires the additional step of showing that when $\underline{\beta}_{h} F_{h}^{N}=h$, we can find a $F_{h}^{T}$ so

\footnotetext{
${ }^{19}$ Recall our simplifying assumption that if the firm fixes the harm is alleviated and there is not fine. Recall also our simplifying assumption that the firm can not fix if it has not investigated since it does not know the nature of the harm.
} 
that $m_{h} \equiv \min \left\{F_{h}^{T}, \bar{\beta}_{h} F_{h}^{N}\right\}=h$. It turns out that $F_{h}^{T}=h$ does the trick: since $\bar{\beta}_{h}>\underline{\beta}_{h}$, setting $\underline{\beta}_{h} F_{h}^{N}=h$ insures that $\bar{\beta}_{h} F_{h}^{N}>h$ and so $F_{h}^{T}=h$ will be the minimum fine defining $m_{h}$.

This is the arithmetic of the solution, but what is going on economically? Intuition is gained by returning to the case of fines $F_{h}$ conditioned on neither disclosure nor investigation, as in subsection 3.2. There we faced the following difficulty: equating the expected fine conditional on investigation with actual harm-in order to set proper post-investigation fixing incentivescauses expected fines conditional on not investigating to fall short of what is necessary to align investigation incentives. Now focus on the mirror image view of this same problem: setting the fine to get the non investigation case right (setting $\underline{\beta}_{h} F_{h}=h$ ) causes expected fines conditional on having investigated to exceed the proper level (i.e., causes $\bar{\beta}_{h} F_{h}>h$ ). The benefit of conditioning on disclosure is that it provides a way of fixing this overshoot in all cases in which the firm has investigated. Essentially, the agency offers the firm who has investigated an "out:" if the firm tells what it's found, the agency lowers the fine below what the firm expects to pay if it keeps quiet. Of course, the firm prefers lower fines, and so always takes the option. In the end, then, the firm faces a post-investigation fine $F_{h}^{T}$ which is lower than that calculated with $F_{h}$ and the higher probability $\bar{\beta}_{h}$. In sum, our verifiability assumption insures that disclosure is only possible when investigation has actually occurred. Conversely, lowering the fine for disclosed violations insures that firms that investigate always disclose. The result is that investigation and disclosure are effectively equated. Hence, the agency's "disclosure discount" allows it to condition fines on investigation per se, which as we saw in subsection 3.4 is the key to setting proper incentives. 


\section{REFERENCES}

Arlen, J., (1994), "The Potentially Perverse Effects of Corporate Criminal Liability," Journal of Legal Studies, 23:833.

and R. Kraakman, (1997) "Controlling Corporate Misconduct: An Analysis of Corporate Liability Regimes," New York University Law Review, 72:687-779.

Banks, J. T., (1996), "EPA's New Enforcement Policy: At Last a Reliable Roadmap to Civil Penalty Mitigation for Self-Disclosed Violation," ELR News \& Analysis 20: 10277-10238.

Cooney, J. F., J. W. Starr, J. G. Block and T J. Kelly, (1995), “Criminal Enforcement of Environmental Laws: Part I," ELR News \& Analysis 25: 10459-10477.

(1995a), "Criminal Enforcement of Environmental Laws: Part II: The Knowledge Element in Environmental Crimes," ELR News \& Analysis 25: 10525-10537.

(1995b), "Criminal Enforcement of Environmental Laws: Part III: From Investigation to Sentencing and Beyond," ELR News \& Analysis 25: 10600-10607.

Davidson, J. (1995c). "Privileges for Environmental Audits: Is Mum Really the Word?" South Carolina Environmental Law Journal 4:111.

Elliott E. D., M. T. Morley and H. L. Pitt, "A Practical Guide to Writing Environmental Disclosures," ELR News \& Analysis 25: 10237-10243.

Environmental Protection Agency ("EPA"), "Incentives for Self-Policing: Discovery, Disclosure, Correction and Prevention of Violations," 60 Fed. Reg. 66,706 (1995).

Feeley, M. S. (1995), "Dirty Secrets: California Moves to Protect Businesses that Conduct Environmental SelfAudits," The Los Angeles Daily Journal July 13:

Kirsch, L. S. and J. W. Viers, (1996) "Environmental Law," The National Law Journal, March 11: B5-B6.

Moore, J. and N. Newkirk (1995), "Not Quite a Giant Step," The Environmental Forum, May/June: 16-21.

Murray, P. C. (1995). "From the Environment: The Environmental Self-Audit Privilege," Real Estate Law Journal 24:169.

Polinsky, A. and D. Rubinfeld (1988), "The Welfare Implications of Costly Litigation for the Level of Liability," Journal of Legal Studies, 17:151-164.

Shavell, S. (1980), "Strict Liability versus Negligence," Journal of Legal Studies, 9:1. 
1997-1998 Discussion Paper Series

\author{
Department of Economics \\ Collumbra Unitersity \\ 1022 International Affairs Bldg. \\ 4207 est 118 th S $S_{\text {treet }}$ \\ New York, N.Y., 10027
}

The following papers are published in the 1997-98 Columbia University Discussion Paper series which runs from early November to October 31 of the following year (Academic Year).

The following is a website address where viewers may download some discussion papers. It is a project that is in progress, and therefore the site may not contain all recent working papers.

http://www.columbia.edu/dlc/wp/econ/index.html.

\title{
For Ordering Hardcopies:
}

Domestic orders for discussion papers are available for purchase at the cost of $\$ 8.00$ (U.S.) Per paper and $\$ 140.00$ (US) for the series.

Foreign orders cost $\$ 10.00$ (US) per paper and $\$ 185.00$ for the series.

To order discussion papers, please write to the Discussion Paper Coordinator at the above address along with a check for the appropriate amount, made payable to Department of Economics, Columbia University. Please be sure to include the series number of the requested paper when you place an order. 
1997-98 Discussion Papers

Economics Department, Columbia University

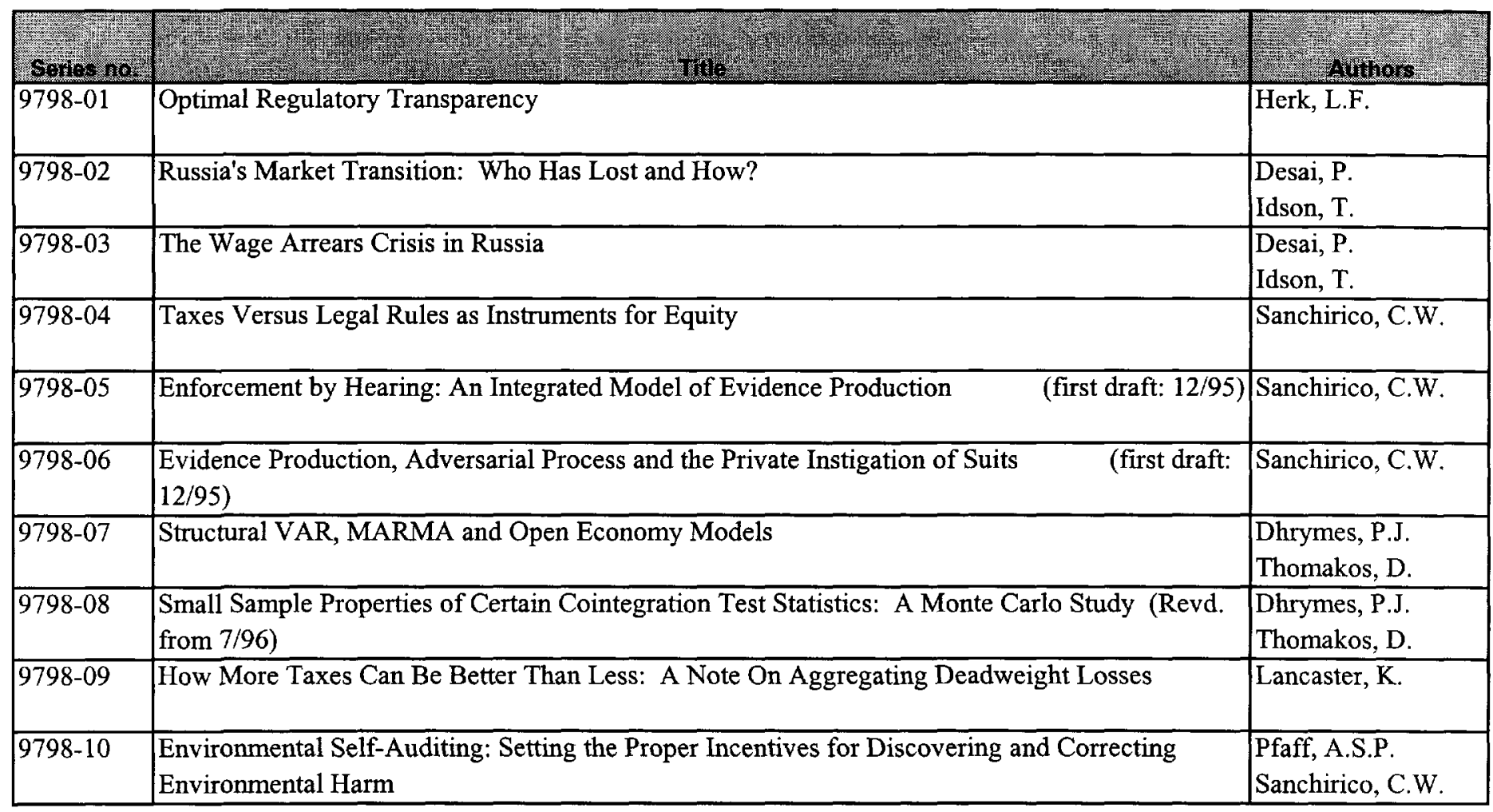

